\title{
Chapter 37 \\ Sperm Proteasome as a Putative Egg \\ Coat Lysin in Mammals
}

\author{
Edward Miles and Peter Sutovsky
}

\begin{abstract}
During animal and human fertilization, the fertilizing spermatozoon creates and passes through a fertilization slit in the vitelline coat (VC) of the oocyte. It has been hypothesized that the penetration of the mammalian $\mathrm{VC}$, the zona pellucida (ZP), is aided by a proteolytic enzyme capable of locally degrading ZP proteins. This putative "zona lysin" is predicted to reside within the sperm head acrosome and be released or exposed by ZP-induced acrosomal exocytosis. Evidence has been accumulating in favor of the $26 \mathrm{~S}$ proteasome, the ubiquitin-dependent multi-subunit protease acting as the putative vitelline coat/zona lysin in humans and animals. To confirm this hypothesis, three criteria must be met: (1) the sperm receptor on the ZP must be ubiquitinated, (2) proteasomes must be present, exposed, and enzymatically active in the sperm acrosome, and (3) sperm proteasomes must be able to degrade the sperm receptor on the egg coat/ZP during fertilization. This review discusses recent data from a number of mammalian and nonmammalian models addressing these predictions. These data shed light on the mechanisms controlling sperm interactions with VC and on the evolutionary conservation of the proteasome-assisted fertilization mechanisms.
\end{abstract}

Keywords Egg coat lysin • Fertilization • Oocyte • Proteasome - Proteolysis

- Sperm $\bullet$ Ubiquitin $\bullet$ Vitelline coat $\bullet$ Zona pellucida

Division of Animal Science and Departments of Obstetrics Gynecology and Women's Health,

University of Missouri, Columbia, MO 65211-5300, USA

e-mail: SutovskyP@missouri.edu 


\subsection{Introduction: Zona Lysin and the Elusive Mechanism of Egg Coat Penetration by Sperm}

The process of mammalian fertilization has long been studied, but many fundamental questions still remain unanswered. One that continues to be debated, despite decades of research, concerns the mechanism utilized by the fertilizing spermatozoa to penetrate the oocyte vitelline coat (VC), the zona pellucida (ZP). When a capacitated, fertilization-competent spermatozoon binds to the sperm receptor on the ZP, it undergoes acrosomal exocytosis (AE), which causes vesiculation of the acrosomal membrane and exocytosis of the acrosomal cap (Yurewicz et al. 1998; Bleil and Wassarman 1980). This step exposes the acrosome-borne proteolytic enzymes and results in the formation of the acrosomal shroud, which allows the spermatozoa to create a local microenvironment that supports the opening of the fertilization slit and penetration of the sperm through the ZP (Yurewicz et al. 1998). There are two schools of thought about the mechanism of sperm-ZP penetration: (1) the mechanical force of the sperm tail motility is sufficient to fully penetrate the ZP (Green and Purves 1984; Bedford 1998); and (2) an enzyme originating from the acrosome of the fertilizing spermatozoa acts as a putative zona lysin that digests the fertilization slit in the ZP and allows sperm to penetrate through the ZP (Austin and Bishop 1958; Austin 1975). The latter theory was first proposed as early as 1958 by Austin and Bishop (1958).

Several candidates have been proposed for this hypothetical zona lysin. Originally, the acrosomal protease acrosin was favored as the most likely zona lysin, but was ruled out when acrosin knockout mice remained fertile with only delayed ZP penetration (Baba et al. 1994). Acrosin has since been considered to be involved in proteolysis or processing of proteins in the acrosome and on acrosomal membranes (Honda et al. 2002). The serine protease Tesp5/Prss21 on the mouse sperm surface was identified as a plausible candidate. Double-knockout studies have shown reduced fertility in vitro, which was rescued by exposure of the spermatozoa to the uterine microenvironment or by treatment with uterine fluids (Yamashita et al. 2008; Kawano et al. 2010). These candidates have since been proposed to be involved in initial sperm-ZP binding (Yamashita et al. 2008; Kawano et al. 2010). Evidence has been accumulating in favor of the $26 \mathrm{~S}$ proteasome as the candidate zona lysin in mammals, ascidians, and invertebrates (Sakai et al. 2004; Yi et al. 2007a; Sutovsky et al. 2004). This review highlights the significant studies and current research examining the possible role of the $26 \mathrm{~S}$ proteasome as the putative mammalian zona lysin.

\subsection{Ubiquitin-Proteasome System}

\subsubsection{Ubiquitin}

Through a multistep enzymatic process, the ubiquitin-proteasome system (UPS) tags outlived or damaged intracellular proteins with a small chaperone protein ubiquitin. This process, referred to as ubiquitination, typically targets the ubiquitinated 
substrates for proteolytic degradation by a multi-subunit protease, the $26 \mathrm{~S}$ proteasome, (Glickman and Ciechanover 2002). Ubiquitin, an 8.5-kDa, 76-amino-acid protein, was first isolated and purified by the Goldstein laboratory in 1975. They found that this polypeptide induced the differentiation of bovine thymus-derived and bone marrow-derived immunocytes in vitro (Goldstein et al. 1975). Consequently, they named this newly discovered polypeptide ubiquitous immunopoietic polypeptide (UBIP) because of its high degree of evolutionary conservation, exhibiting close structural, functional, and immunological similarity when isolated from species as diverse as protozoans to mammals and plants. In 1976, Etlinger and Goldberg first described a novel ATP-dependent proteolytic system responsible for the rapid degradation of abnormal proteins separate from the lysosomal degradation pathway (Etlinger and Goldberg 1977). The discovery that metabolic energy is required for intracellular protein degradation opposed the commonly accepted idea that cellular proteolysis was an entirely exergonic process occurring in the lysosome. In a joint effort, Aaron Ciechanover, Avram Hershko, and Ervin Rose described the ubiquitin-mediated protein degradation, a discovery for which they shared the Nobel Prize in Chemistry in 2004. Ubiquitin is not just a housekeeping protein that helps recycle outlived or damaged protein molecules. It is also involved in a number of cellular mechanisms and pathologies, including but not limited to antigen presentation in the immune system, apoptosis, Alzheimer's disease, cell-cycle control, endocytosis of membrane receptors, human immunodeficiency virus (HIV) particle internalization, protein quality control in the endoplasmic reticulum, reticulocyte differentiation, signaling, and transcriptional control (Pines 1994; Hershko and Ciechanover 1998; Glickman and Ciechanover 2002).

\subsubsection{Ubiquitination}

Ubiquitin is typically a cytoplasmic and nuclear protein, but it can be found in the extracellular space in some mammalian, lower vertebrate, and invertebrate systems and is highly substrate specific. Polymerization of substrate-bound ubiquitin molecules into multi-ubiquitin chains serves as a degradation signal for numerous target proteins. The degradation of substrate protein is initiated by the covalent attachment of an isopeptide chain of four or more ubiquitin molecules. These ubiquitin molecules are linked to each other through one of seven lysine residues (K6, K11, K27, K29, K33, K48, K63) to form the poly-ubiquitin chains. All seven of these Lys residues are potential ubiquitin-chain initiation sites, but $\mathrm{K} 48$ is the most common linkage site for poly-ubiquitin chains recognized by the 26S proteasome (Walczak et al. 2012; Iwai 2012). The K63 site is the most common site of di-ubiquitination, targeting membrane receptors for lysosomal degradation, chromatin remodeling, and DNA repair (Walczak et al. 2012; Kim et al. 2006).

The formation and ligation of the poly-ubiquitin chain to its target protein occurs through a concerted series of ATP-dependent enzymatic reactions (Fig. 37.1). Ubiquitination starts with the activation of a single ubiquitin molecule with a 


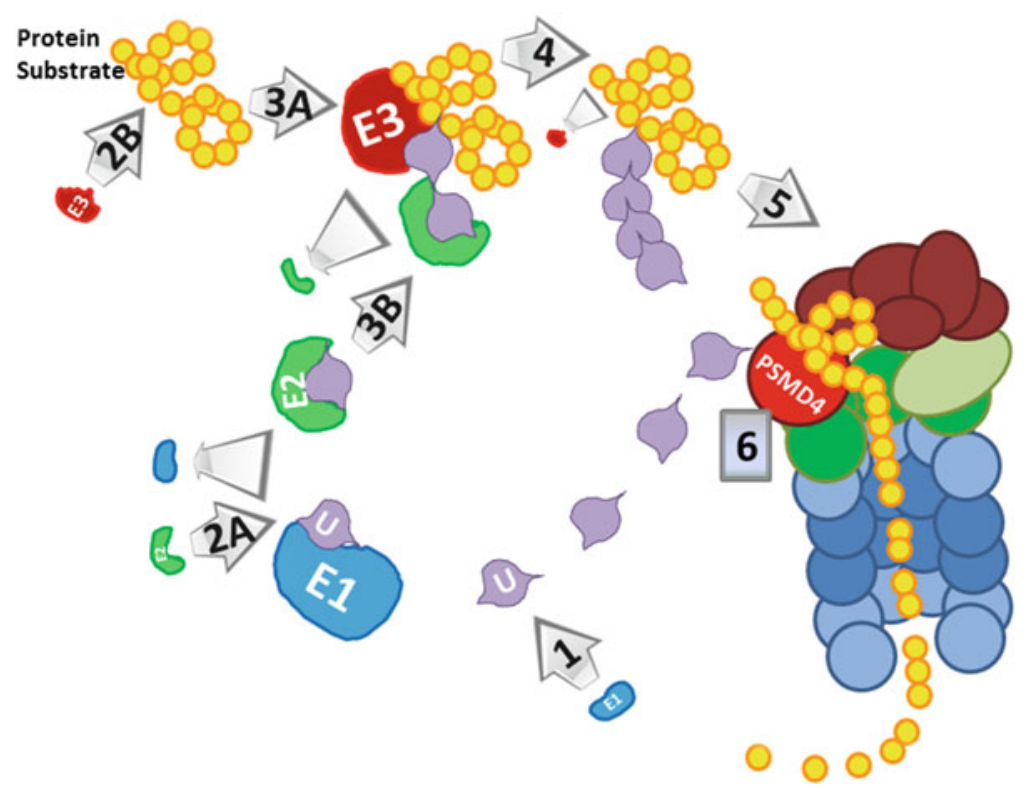

Fig. 37.1 Diagram of protein ubiquitination and degradation by the $26 \mathrm{~S}$ proteasome. Step 1 . Monoubiquitin is activated by a phosphorylated ubiquitin-activating enzyme E1 (UBA1). Step $2 A$. UBE1 is supplanted by ubiquitin carrier enzyme/ubiquitin-conjugating enzyme E2 (UBE2). Step $2 B$. Concurrently, the substrate protein is engaged by an E3-type ubiquitin ligase. Step $3 A$. Ubiquitin ligase E3 covalently links an activated monoubiquitin to an internal Lys residue of the substrate protein. Step $3 B$. A second activated ubiquitin molecule is linked to the substrate-bound ubiquitin. Step 4 . The ensuing tandem ligation of additional activated ubiquitin molecules results in the formation of a multi-ubiquitin chain. Step 5. Multi-ubiquitin chain of four or more ubiquitin molecules is recognized and engaged by subunit PSMD4 of the 19S proteasomal regulatory complex. Step 6. The substrate protein is deubiquitinated (liberated ubiquitin molecules re-enter the cycle), unfolded, threaded through the $20 \mathrm{~S}$ core, and cleaved into small peptides, released from the 20 S core lumen. [From Sutovsky 2011, (C) Society for Reproduction and Fertility (2013). Reproduced by permission]

phosphorylation-dependent ubiquitin-activating enzyme E1 (UBE1 or UBA1 in HUGO nomenclature). The UBA1 is then supplanted by ubiquitin carrier/ubiquitinconjugating enzyme E2. Simultaneously, an E3-type ubiquitin ligase seeks out and engages the target protein to be ubiquitinated and degraded (Hershko 2005). The E3-type ligases are highly diverse and responsible for the substrate specificity of protein ubiquitination as there are about two E1 proteins, approximately 30 E2 proteins, and more than 500 different E3 proteins in humans (Tanaka 2009). The E3-ligases catalyze the covalent ligation of the C-terminal glycine/Gly residue (G76) of ubiquitin to an internal lysine/Lys residue of the target protein. Next, the ubiquitin chain elongates from the mono-ubiquitinated substrate protein resulting in a poly-ubiquitinated protein. A poly-ubiquitin tail of four or more ubiquitin molecules is needed to signal proteasomal degradation. 

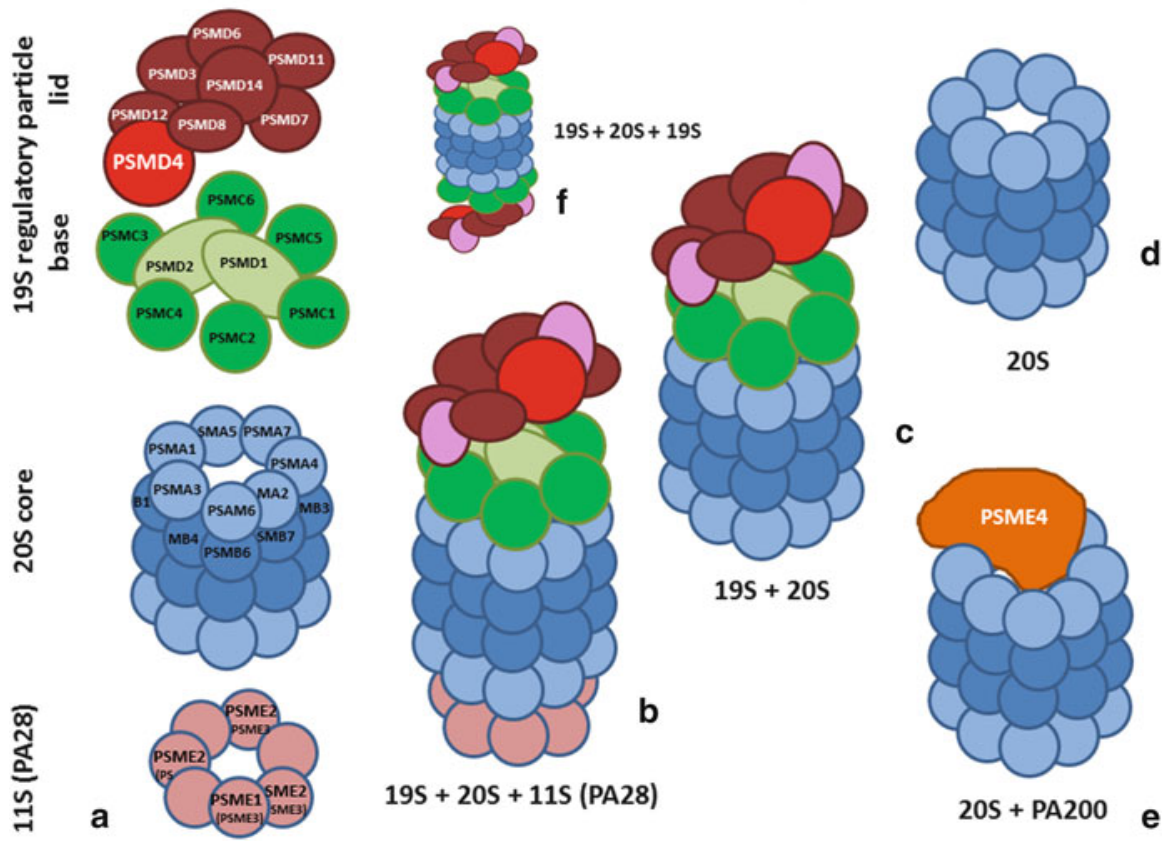

Fig. 37.2 Variations on the subunit composition of the 26S proteasome. (a) Subunit makeup of (top to bottom) $19 \mathrm{~S}$ regulatory particle (lid + base), 20S core, and 11S particle (PA28). (b) 20S core capped with $19 \mathrm{~S}$ particle on one side and an $11 \mathrm{~S}$ complex/PA28 on the other. (c) $20 \mathrm{~S}$ core capped with one 19S particle. (d) Uncapped 20S proteasome. (e) 20S core capped with proteasome activator PA200. (f) Canonical $26 \mathrm{~S}$ proteasome, with one $19 \mathrm{~S}$ particle/cap on each side of $20 \mathrm{~S}$ core. [From Sutovsky 2011, (C) Society for Reproduction and Fertility (2013). Reproduced by permission]

\subsubsection{S Proteasome}

The $26 \mathrm{~S}$ proteasome is a $2.5-\mathrm{MDa}$ multi-catalytic canonical protease localized in the cell cytosol and nucleus (Fig. 37.2). The 26S proteasome is responsible for degrading ubiquitinated proteins in the cell, although nonubiquitinated proteins could be substrates as well. The typical enzymatically active proteasome consists of two subcomplexes. One is a hollow catalytic 20S core particle (20S CP) capped by one or two terminal 19S regulatory particle(s) (19S RP). The 19S RP is responsible for poly-ubiquitin chain recognition and binding, deubiquitination, and substrate protein priming/linearization/unfolding. The 19S RP consists of at least 17 different subunits between the two subcomplexes: the lid and the base. The lid complex contains 14 regulatory particle non-ATPase subunits (PSMD1-14) (Hanna and Finley 2007). Subunit PSMD4 (Rpn10) is the main 19S subunit responsible for substrate recognition. It binds the poly-ubiquitin chains on the target protein during polyubiquitin chain recognition by the $26 \mathrm{~S}$ proteasome (Yi et al. 2010a, b). Subunit PSMD14 (Rpn11), then deubiquitinates the captured substrate by cleaving the 
poly-ubiquitin chain at a proximal site, which is then further cleaved into single ubiquitin molecules by deubiquitinases (DUBs) that can associate with the 19S RP (Verma et al. 2002). The cleaved ubiquitin molecules can be reused for ubiquitination of other protein molecules. New functions are still being discovered for subunits composing the 19S lid. Table 37.1 lists the known 26S proteasomal subunits and their proposed functions by species.

The 19S RP base subcomplex is responsible for (1) capturing target proteins via poly-ubiquitin chain recognition, (2) unfolding the substrate protein, and (3) opening the channel into the 20S CP. The base subcomplex contains six homologous AAA-ATPase subunits (PSMC1-6) and three non-ATPase subunits (PSMD1, -2, -4). These subunits create a narrow gated channel, which only allows unfolded proteins to enter the 20S core. PSMD1 and -2 have been proposed to work together as a functional unit and are required for substrate translocation and opening of the $20 \mathrm{~S}$ channel. This compartmentalized design of the $26 \mathrm{~S}$ proteasome separates proteolysis from the cellular milieu and restricts degradation to unfolded and imported proteins. Subunit PSMD2 attaches to the 20S CP, whereas subunit PSMD1 is located on top of PSMD2 and serves as a docking site for substrate recruitment factors (Rosenzweig et al. 2008). Subunit PSMD4 functions as an integral ubiquitin receptor to trap poly-ubiquitinated proteins; PSMD4 does this by its C-terminal ubiquitininteracting motif (Deveraux et al. 1995). Six ATPase subunits (PSMC1-6) surrounding the base are organized into a hexameric ring that controls the opening of the channel and allows the protein to reach the catalytic sites of the 20S CP. The base ATPases are required not only to open the channel into the 20S CP but to also unfold the substrate protein: this allows the protein to be threaded through the narrow channel of the 20S CP where the catalytic protease subunits are located. The details of the ATP-dependent mechanisms behind these processes are still unknown, but it is certain that these subunits work through a process that requires ATP hydrolysis (Liu et al. 2006).

The 20S CP is a 750-kDa cylindrical protein complex responsible for the proteolysis of the substrate protein. The 20S CP contains 28 subunit molecules of 14 types formed from the stacking of two outer $\alpha$-rings and two inner $\beta$-rings. Each ring is composed of seven structurally similar $\alpha$ - and $\beta$-subunits, respectively. The rings are heptamerically stacked in an $\alpha_{1-7} \beta_{1-7} \beta_{1-7} \alpha_{1-7}$ pattern. The subunits of the $\alpha$-ring (PSMA1-7) connect the 20S CP to the 19S base and act as a gate that opens in the presence of an ubiquitinated protein. The two inner rings are each composed of seven $\beta$-type subunits (PSMB1-7) and confer the threonine protease activities of the $26 \mathrm{~S}$ proteasome. There are three catalytic $\beta$-type subunits: PSMB6 ( $\beta 1)$, PSMB7 $(\beta 2)$, and PSMB5 ( $\beta 5)$. The $\beta 1$-subunit is associated with caspase-like/PGPH (peptidylglutamyl-peptide hydrolyzing) activity, $-\beta 2$ with trypsin-like activity, and $\beta 5$ with chymotrypsin-like activities, which confer the ability to cleave peptide bonds at the C-terminal side of acidic, basic, and hydrophobic amino-acid residues, respectively (Tanaka 2009). The 20S proteasome then degrades the target protein into 3 to 15 amino-acid oligopeptides that are released into the cytosol and are further hydrolyzed into single amino acids by cytosolic oligopeptidases or amino-carboxyl peptidases (Tanaka 2009). 
37 Sperm Proteasome as a Putative Egg Coat Lysin in Mammals

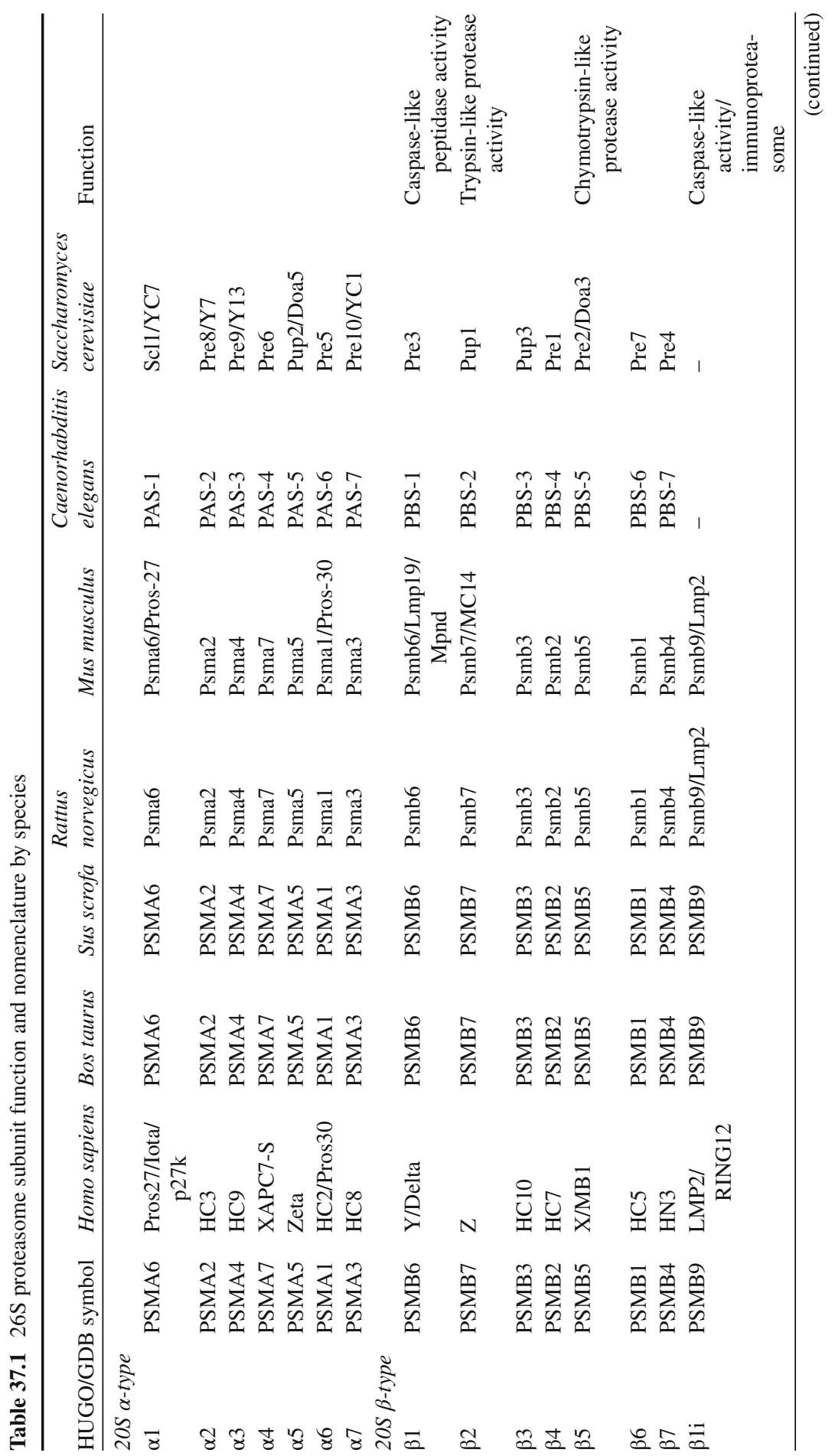




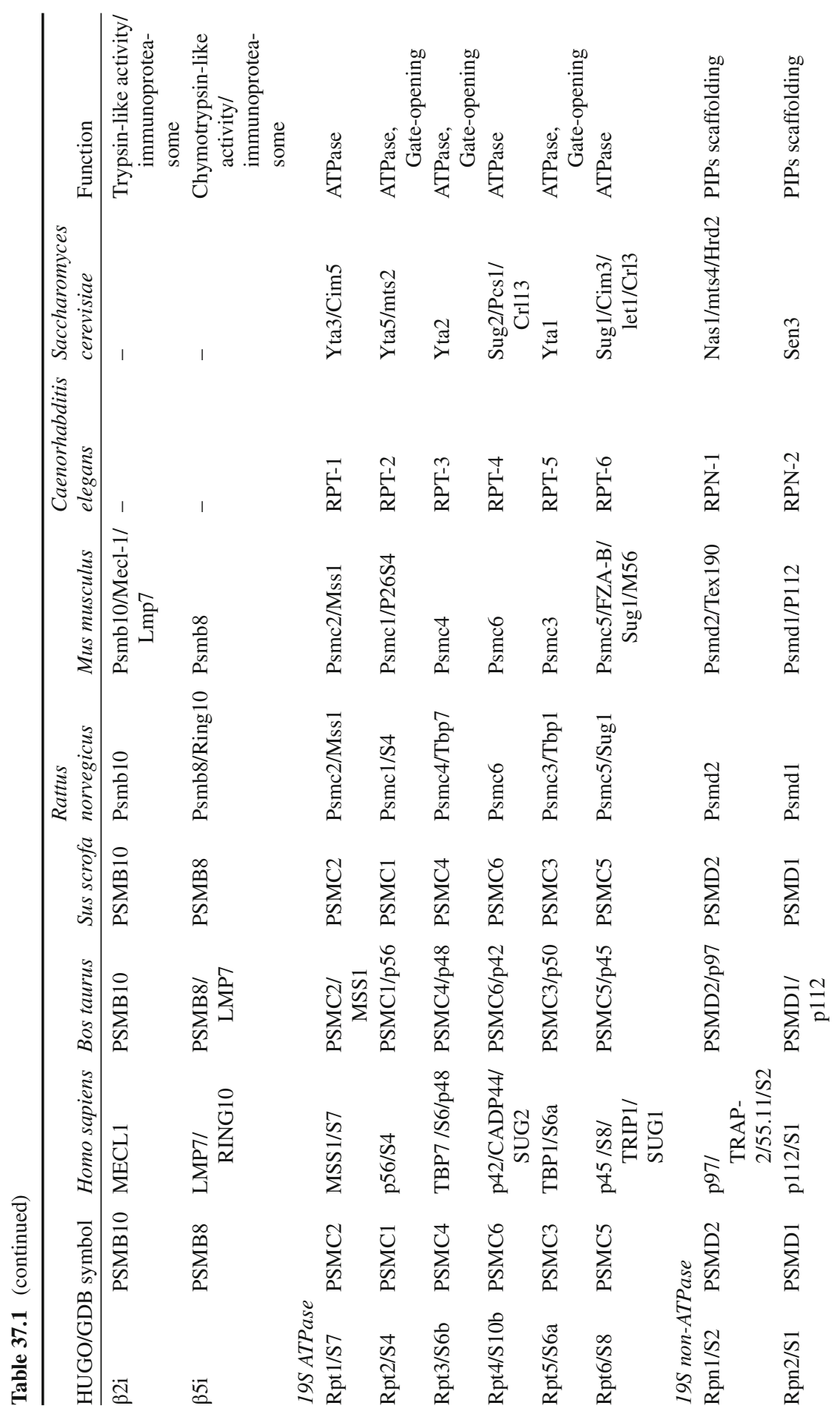




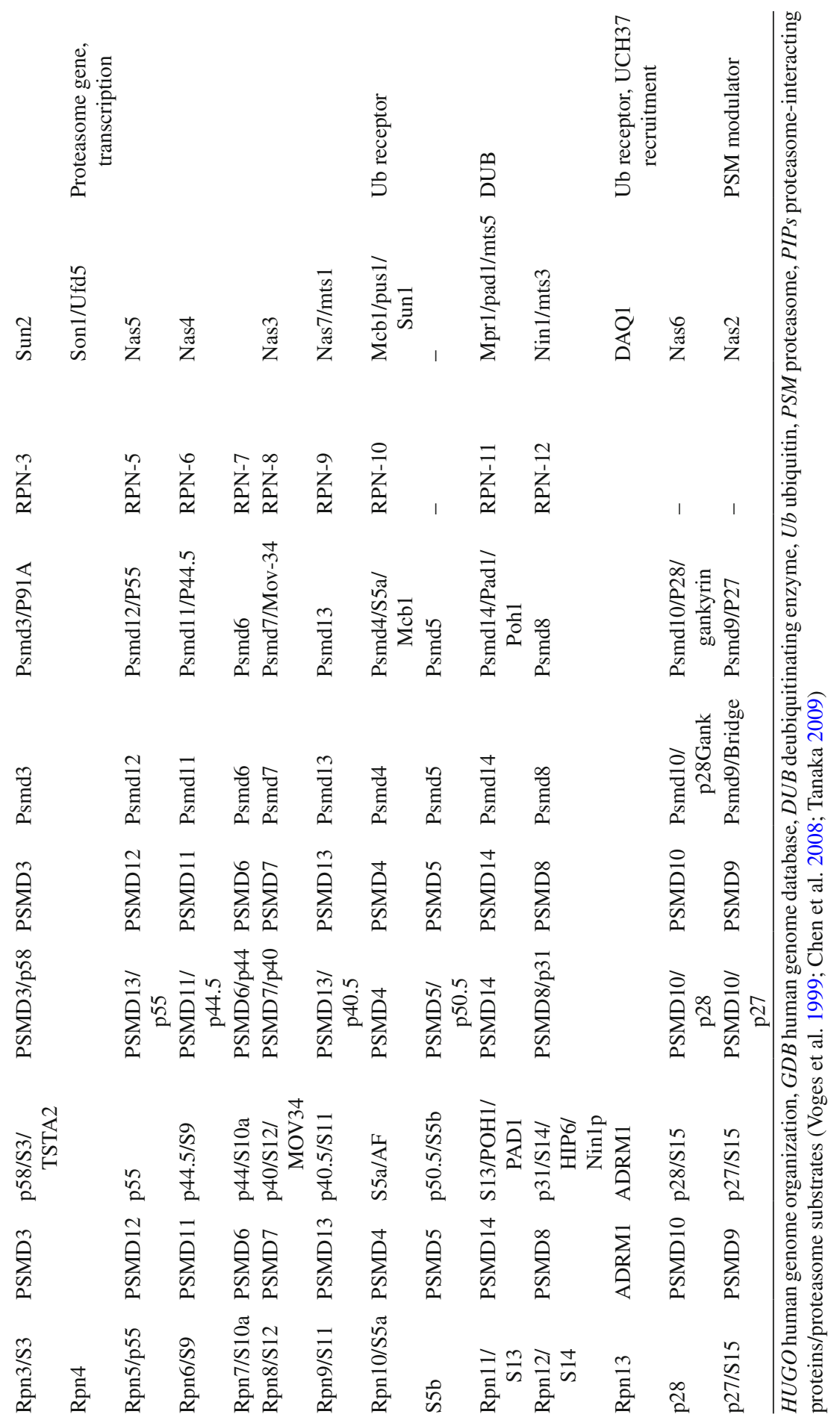




\subsection{Sperm Proteasome During Plant, Ascidian, and Echinoderm Fertilization}

\subsubsection{Plant Fertilization}

The sexual reproduction cycle in higher plants occurs in the reproductive organs of the flower. Similar to animal spermatozoon, pollen, the male gametophyte, is a highly specialized structure, consisting of two or three cells. Pollen is released from the anthers and adheres to the surface of the female stigma, equipped with a cytoplasmic extension known as the pollen tube (Boavida et al. 2005). The pollen tube rapidly grows through the style to the ovules (Boavida et al. 2005). Pollen tube adhesion and growth guides the pollen toward the ovary where it fertilizes the embryo sac, the female gametophyte (Boavida et al. 2005). For a more in-depth account of fertilization in higher plants, please refer to the review by Boavida et al. (2005).

Evolutionary conservation of the components and roles of the UPS appear to reach beyond the animal kingdom to plants, where pollen adhesion and guidance seem to depend on ubiquitin. For example, during maize pollen development, the levels of ubiquitin and ubiquitin-protein conjugates in young pollen precursors, the microspores without vacuoles were 10 to 50 times lower than compared to mature pollen grains (Callis and Bedinger 1994). Treatment with $26 \mathrm{~S}$ proteasomal inhibitors, MG132 and epoxomicin, significantly reduced pollen tube growth in kiwifruit (Actinidia deliciosa) (Speranza et al. 2001). Kim et al. (2006) showed that pollen adhesion to styles of lily (Lilium longiflorum Thunb.) can be enhanced in vitro with the addition of exogenous ubiquitin. Furthermore, in Arabidopsis thaliana the proper conformation of SCF E3 ubiquitin-ligases that direct ubiquitination is required for male fertility (Devoto et al. 2002). Mutations in genes encoding 26S proteasomal subunits, ubiquitin-specific proteases, deubiquitinating enzymes, and $19 S$ regulatory particle non-ATPases result in infertility from defects in pollen maturation or transmission (Doelling et al. 2007; Book et al. 2009). Several E3s have been shown to play key roles in self/non-self pollen recognition in snapdragons (Antirrhinum) (Qiao et al. 2004). In snapdragons, the SCF ${ }^{\text {AhSLF2 }}$ complex acts as an E3 that targets non-self S-RNases for ubiquitination and destruction by the $26 \mathrm{~S}$ proteasome; however, it leaves self-S-RNases active during the self-incompatibility response, thus functioning as a cytotoxin that degrades RNA and terminates pollen tube growth (Qiao et al. 2004). The current data strongly suggest a role for the UPS in plant germination and gametophyte development, but more research is needed to elucidate how the UPS is utilized in these processes.

\subsubsection{Ascidian Fertilization}

Ascidians are hermaphrodites that reproduce by releasing sperm and eggs simultaneously into the surrounding seawater during the spawning season; thus, large 
quantities of readily fertilizable gametes can be easily obtained for research. The exposure to alkaline seawater activates the spermatozoa, very similar to capacitation in mammals, suggesting that the proteasome is then secreted to the sperm surface. The spermatozoa then undergo species-specific binding to the proteinaceous vitelline coat, which they must penetrate via sperm surface proteases that act as a VC-lysin to complete the fertilization process. It has been shown that the proteasomes are exposed on the ascidian sperm surface; proteasomal proteolytic activity was specifically detected in the sperm head region and was clearly increased upon sperm activation (Sawada et al. 2002a, b). Anti-proteasome antibodies, and proteasomal inhibitors MG115 and MG132, inhibit ascidian fertilization (Sawada et al. 2002b).

Studies in two ascidian species, Ciona intestinalis and Halocynthia roretzi, have shown a strict self-sterility in fertilization (Sawada et al. 2002a). After self/non-self recognition via interactions between the sperm and $\mathrm{VC}$, the sperm-borne egg coat lysin system is activated (Sawada et al. 2002a). Data from Sawada's laboratory have provided evidence that $\mathrm{VC}$ penetration by ascidian sperm involves the sequential ubiquitination and proteasomal degradation of the sperm receptor protein $\mathrm{HrVC70}$, a major protein component of the ascidian VC. Ubiquitination of $\mathrm{HrVC70}$ is accomplished by a 700-kDa ubiquitin-conjugating enzyme complex released during sperm activation (Sawada et al. 2002a; Sakai et al. 2003). A 930-kDa proteasome (26S-like proteasome) seems to function as the egg coat lysin that directly degrades the VC. These data suggest that an extracellular, sperm-borne, ubiquitin-conjugating enzyme system is essential for the formation of these poly-ubiquitin chains on HrVC70 and for ascidian fertilization.

The HrVC70 is a 70-kDa component of the VC shown to be a novel sperm receptor that bears significant similarity to components of the mammalian zona pellucida, particularly the proposed mammalian/murine sperm receptor ZPC (Sawada et al. 2002a). Three N-terminal cystine/Cys residues in the ZP domain of the 120-kDa HrVC70 precursor (HrVC120) share conserved positions within mammalian and frog ZPC (Sawada et al. 2002a). HrVC70 contains 12 EGF (epidermal growth factor)-like repeats that confer its role in sperm binding to VC (Sawada et al. 2002b). This protein has been shown to be degraded by purified ascidian sperm 26S-like proteasomes only in the presence of ubiquitin, ATP, and the ubiquitin-conjugating enzymes purified from a rabbit reticulocyte lysate (Sawada et al. 2002b; Sakai et al. 2004). These results reveal that an extracellular UPS is essential for ascidian fertilization, particularly in the degradation of the proteinaceous vitelline coat.

Recently, Yokota and Sawada investigated an extracellular transport signal and have discovered a novel posttranslational modification of the ascidian sperm proteasome. They found that the 20S core PSMA1/ $\alpha 6$-subunit of purified sperm proteasomes is distinct from purified egg and muscle proteasomes (Yokota et al. 2011). Tissue specific $\alpha$-subunits are not commonplace, but several are expressed in the testis of Drosophila ( $\alpha 3 \mathrm{~T}, \alpha 4 \mathrm{~T} 2$, and $\alpha 6 \mathrm{~T}$ ) (Belote and Zhong 2009). Among these testis-specific $\alpha$-subunits, $\alpha 6 \mathrm{~T}$ is reported to be crucial for spermatogenesis and fertility (Belote and Zhong 2009). The $\alpha 6$-subunit of $H$. roretzi contains a cluster of acidic amino acid residues and the removal of this cluster may mimic the state of dephosphorylation of the $\alpha 6$-subunit which may affect function and localization of 
the sperm proteasome (Yokota et al. 2011). Alternatively, it is also possible that the conserved sequence of the $\alpha 6$-subunit in $H$. roretzi and $C$. intestinalis may function as a transport signal to the acrosome during ascidian spermiogenesis. It is not known at present if similar modifications exist in the mammalian sperm proteasome.

\subsubsection{Echinoderm Fertilization}

Most echinoderms can reproduce sexually by spawning eggs and spermatozoa into the seawater, much as do ascidians, and asexually by regenerating body parts. For the purpose of this review, only sexual reproduction is examined. It has been confirmed that the proteasomes are present in sea urchin spermatozoa and that the sperm proteasome may be involved in the acrosome reaction; but differing from ascidians, the proteasome is not essential for sperm binding to the echinoderm VC (Yokota and Sawada 2007; Matsumura and Aketa 1991). The role of sperm proteasomes in echinoderm fertilization has been investigated in the sea urchin Pseudocentrotus depressus by Yokota and Sawada (2007), who examined the effects of proteasomal inhibitors and synthetic peptide substrates for the proteasome on different steps of the fertilization process. The inhibition of fertilization by proteasomal inhibitors suggests that the echinoderm sperm proteasomes are important in the acrosome reaction and in sperm penetration through the $\mathrm{VC}$, most likely as an egg coat lysin (Yokota and Sawada 2007). Among the examined proteasomal substrates and inhibitors, a caspase substrate (Z-Leu-Leu-Glu-MCA) competitively inhibited the caspase-like catalytic center ( $\beta 1$-subunit) of the proteasome, which decreased fertilization in a concentration-dependent manner (Yokota and Sawada 2007 ); this suggests that the caspase-like activity of the proteasome $\beta 1$-subunit must play a key role in sea urchin fertilization.

Echinoderm spermatozoa undergo acrosome reaction/acrosomal exocytosis when they enter the jelly coat surrounding the VC. Protein kinase A (PKA) has been implicated in the acrosome reaction in sea urchins ( $\mathrm{Su}$ et al. 2005), and shown to stimulate the function of mammalian 26 S proteasome (Zhang et al. 2007). Therefore, the sperm proteasome may act to degrade certain unknown PKA modulators, which would result in the irreversible activation of PKA during the acrosome reaction. During the acrosome reaction, the acrosomal contents containing an egg coat lysin are exposed and released, allowing spermatozoa to penetrate the VC (Yokota and Sawada 2007). For the sperm proteasome to act as egg coat/VC lysin, the VC must be ubiquitinated before fertilization and extracellular ATP must be present (Yokota and Sawada 2007). Yokota and Sawada used Western blotting with a monoclonal anti-ubiquitin antibody FK2, which recognizes both mono- and multi-ubiquitinated proteins, to reveal a band pattern indicative of $\mathrm{VC}$ ubiquitination. The identity of the ubiquitinated VC protein(s) remains to be determined, but current data support the hypothesis that the echinoderm sperm $26 \mathrm{~S}$ proteasome is released during the acrosome reaction and degrades the ubiquitinated VC proteins in an ATP-dependent fashion. 


\subsection{Proteasome Localization and Activity in Mammalian Spermatozoa}

Mammalian fertilization is a unique cellular-recognition event that comprises sequential interactions between the sperm and the oocyte vitelline coat (Yanagimachi 1994). First, a capacitated spermatozoon penetrates the cumulus oophorus, facilitated by a sperm membrane-bound hyaluronidase, and then binds to the zona pellucida, a protective glycoprotein matrix that surrounds the ovulated oocyte. The ZP plays a pivotal role in the species specificity of sperm-oocyte recognition, and binding, as well as in the induction of acrosomal exocytosis (AE), anti-polyspermy defense, and protection of the embryo until implantation. In most of the mammals studied, only spermatozoa with an intact acrosome bind to the ZP, in a speciesspecific manner. The fertilizing spermatozoan interaction with the zona glycoprotein that serves as sperm receptor (ZP3/ZPC in mouse/human and ZPB/ZPC complex in pig) triggers the AE via signal transduction events in the sperm acrosome, such as the opening of T-type, low voltage-activated $\mathrm{Ca}^{2+}$ channels, causing an influx of $\mathrm{Ca}^{2+}$ and the activation of heterotrimeric $\mathrm{G}$ proteins (Pasten et al. 2005; Ikawa et al. 2010). An increase in intracellular $\mathrm{pH}$, resulting in sustained $\mathrm{Ca}^{2+}$ influx, which drives AE, causes the release or exposure of acrosomal enzymes and, when combined with vigorous sperm motility, allows the spermatozoon to penetrate the ZP and fuse with the oocyte plasma membrane, the oolemma (Pasten et al. 2005; Ikawa et al. 2010).

The role of the UPS has been well documented in the reproductive processes of several mammalian species. For example, multi-enzymatic sperm protease complexes with similar sedimentation coefficients as purified proteasomes from other tissues have been isolated from mouse (Pasten et al. 2005), pig (Zimmerman and Sutovsky 2009), and human (Morales et al. 2003; Morales et al. 2004; Baker et al. 2007) spermatozoa. Accordingly, the substrate-specific enzymatic activities (trypsinlike, chymotrypsin-like, and peptidylglutamyl peptide-hydrolyzing activities) of these sperm proteasomes have been recorded in mice (Pasten et al. 2005; Bedard et al. 2011; Rivkin et al. 2009), pigs (Sutovsky et al. 2004; Yi et al. 2007a; Yi et al. 2009), and humans (Morales et al. 2003; Chakravarty et al. 2008; Kong et al. 2009).

The sperm-associated proteasomes are tethered to the acrosomal surface in mice (Pasten et al. 2005), pigs (Sutovsky et al. 2004; Yi et al. 2009), and humans (Morales et al. 2004). The proteasomes probably become associated with the spermatozoa inner and outer acrosomal membranes during acrosomal biogenesis at the spermatid stage (Rivkin et al. 2009). It appears that the extracellular proteasomes exposed on the acrosomal surface of mammalian spermatozoa are involved in the ZP-induced $\mathrm{AE}$ and are able to directly interact with the ZP during fertilization (Sutovsky et al. 2004; Yi et al. 2009). The proteasomes located on the cell surface could participate in sperm-ZP binding in some mammalian species, such as humans (Chakravarty et al. 2008; Naz and Dhandapani 2010) and mice (Pasten et al. 2005). Then, the intracellular and extracellular proteasomes would blend into the acrosomal shroud and participate during the AE. Sperm proteasomes have been shown to be essential 
for acrosomal function and sperm-zona penetration during fertilization in several mammalian species such as mouse, pig, and human (Pasten et al. 2005; Kong et al. 2009; Zimmerman et al. 2011). It is possible that the proteasomes modulating these cellular events could participate in other steps of fertilization as well.

In addition to proteasomes, other UPS components have been implicated in spermatogenesis, epididymal sperm maturation, and fertilization. For example, ubiquitinated substrates have been detected in the epididymal fluid, the seminal plasma, on the surface of defective epididymal spermatozoa, and on the outer surface of the ZP (Sutovsky et al. 2001, 2004; Zimmerman et al. 2011). Anti-proteasome antibodies have been reported to be present in the seminal plasma of infertile men (Bohring et al. 2001; Bohring and Krause 2003). Addition of the deubiquitinating enzyme inhibitor, ubiquitin-aldehyde, to boar in vitro fertilization assays promotes polyspermy, which might be related to an increase in ubiquitination and degradation of the ZP because this C-terminally modified ubiquitin species accelerates proteasomal proteolysis (Yi et al. 2007b). More recently, sperm deubiquitinating enzymes have been explored by the Wing laboratory in Canada. They reported that the spermatozoa of deubiquitinating enzyme Usp2 knockout mice are severely subfertile, which appeared to be caused by a defect in ZP binding or penetration, even though the USP2 protein in wild-type mice does not seem to be specifically localized in the acrosome. In elongating spermatids, USP2 was localized perinuclearly in a thin layer between the outer acrosomal membrane and the plasma membrane, but absent from the nucleus (Lin et al. 2000). The Usp2 null spermatozoa are motile and undergo the $\mathrm{AE}$ but fail to penetrate the $\mathrm{ZP}$ (Bedard et al. 2011). These data suggest that the deubiquitination of sperm or spermatid proteins by USP2 is an important regulatory mechanism required for the acquisition of fertilizing ability by mammalian spermatozoa. It is not clear if the observed severe subfertility is a result of impaired deubiquitinating activity of the acrosomal USP2 enzyme during fertilization, or if it is rather a result of abnormal assembly of the sperm acrosome during spermiogenesis. Either way, the failure of motile Usp2 null spermatozoa to penetrate the zona supports the view that sperm motility alone is not sufficient to propel the sperm head through the zona matrix.

The role of UPS in sperm penetration through the ZP has been well documented. Proteasomal inhibitors and anti-proteasome antibodies effectively block mouse, pig, and human sperm ability to penetrate the ZP of their respective species (Morales et al. 2003; Sutovsky et al. 2004; Pasten et al. 2005). The coincubation of pig spermatozoa with proteasomal inhibitors prevents sperm-ZP penetration without affecting sperm motility and binding (Sutovsky et al. 2003, 2004; Yi et al. 2007a; Zimmerman and Sutovsky 2009). However, if the zona is removed before fertilization, fertilization proceeds even with the addition of proteasomal inhibitors (Sutovsky et al. 2004), which suggests a role for the proteasome in sperm-ZP penetration. Chakravarty et al. reported that the human sperm-associated proteasomal activity is not stimulated by binding of recombinant $\mathrm{ZP}$ proteins, but that it remains steady after AE, most likely caused by the association of the proteasomes with the inner acrosomal membrane, which is not removed by AE (Chakravarty et al. 2008). 
The ZP3/ZPC in mice and humans has been identified as the primary spermbinding receptor on the $\mathrm{ZP}$ and perhaps the inducer of $\mathrm{AE}$ (Naz and Dhandapani 2010). The human sperm ubiquitin-associated protein UBAP2L was identified as a ZP-interacting protein by the Naz Lab (Naz and Dhandapani 2010) using a yeast two-hybrid screen $(\mathrm{Y} 2 \mathrm{H})$ and was further tested when UBAP2L antibodies were shown to inhibit sperm-ZP binding when tested in vitro. The $\mathrm{Y} 2 \mathrm{H}$ procedure is used to identify proteins that interact with a target protein expressed in yeast as a hybrid with a DNA-binding domain and has been widely used to examine proteinprotein interactions (Naz and Dhandapani 2010). Human sperm-oocyte recognition, binding, and $\mathrm{AE}$ have been proposed to be mediated by sugar residues $(\mathrm{O}$ - and $\mathrm{N}$-linked) and peptide moieties of ZP3 (Chakravarty et al. 2005; Gupta et al. 2009). Results from the Naz lab suggest that ubiquitination, in addition to glycosylation of the ZP proteins, may regulate the sperm-ZP interactions (Naz and Dhandapani 2010).

In agreement with the foregoing study, Aitken and colleagues found that the proteasome is a component of a multimeric ZP-binding complex found in human spermatozoa (Redgrove et al. 2011). They propose that sperm-ZP binding requires the concerted action of several sperm proteins that form multimeric recognition complexes on the sperm surface, which is an alternate and novel view different from the traditional simple lock-and-key mechanism of one receptor, one ligand. The formation of these complexes on the sperm surface is purported to depend upon posttesticular maturation driven by the environmental changes to which the spermatozoa are exposed in the epididymis and within the female reproductive system. They report that human spermatozoa express a number of high molecular weight protein complexes on their surface and that subsets of these complexes display affinity for homologous ZP (Redgrove et al. 2011). Two of these complexes were revealed to be a chaperonin-containing TCP-1 (CCT), which harbors a putative ZP-binding protein, ZPBP2, and several components of the $20 \mathrm{~S}$ proteasome that were found previously in an analysis of the human sperm proteome (Redgrove et al. 2011; Johnston et al. 2005). The role of these protein complexes in sperm-ZP interactions was further confirmed when antibodies against the individual components of the complex, including proteasomal subunits, inhibited sperm binding to zonaintact oocytes (Redgrove et al. 2011). Because many sperm proteins exhibit ZP-binding affinity, it is possible that complexes containing these ZP-binding proteins may participate in sequential or hierarchical molecular interactions or act synergistically to ensure successful sperm-ZP binding. It is important to note that the proteasome complex was shown as three large bands when examined by blue native polyacrylamide gel electrophoresis. Aitken et al. theorize that this is caused by posttranslational modifications of certain subunits of the complex ( $\alpha 1-7, \beta 1$, and $\beta 4)$ that displayed charge shift signatures characteristic of tyrosine phosphorylation (Redgrove et al. 2011). This finding is consistent with the proteasomal subunit phosphorylation reported in the acrosome that, according to Diaz et al., modulates the fertilizing capacity of human spermatozoa by inducing AE (Diaz et al. 2007), suggesting that proteasome complexes may be differentially activated during the individual steps of fertilization. 


\subsection{Is the Mammalian Egg Coat Ubiquitinated Before Fertilization?}

To confer with the hypothesis that the $26 \mathrm{~S}$ proteasome acts as the putative egg coat lysin in mammalian fertilization, there must be an ubiquitinated sperm receptor on porcine ZP that is degraded by the sperm acrosome-borne proteasomes during porcine fertilization. These three prerequisites are required: (1) the sperm receptor on the mammalian egg coat is ubiquitinated; (2) proteasomes are present, exposed, and enzymatically active in the sperm acrosomal cap; and (3) sperm proteasomes degrade the sperm receptor on the egg coat during fertilization.

There is evidence that the sperm receptor complex ZPB-ZPC on the porcine ZP is ubiquitinated. In porcine oocytes, the ZPB-ZPC complex has been shown to be responsible for sperm-ZP binding (Yurewicz et al. 1998). Furthermore, the porcine ZPC homologues in mouse (ZP3) and human (ZP3) have been implicated in sperm binding and induction of acrosomal exocytosis (Shur et al. 2006; Gupta et al. 2009). Sutovsky et al. (2004) have shown the presence of ubiquitinated proteins in the unfertilized porcine egg coat. They reported that ubiquitin-immunoreactive proteins can be detected on the outer surface of porcine ZP, visualized ZP digestion with immunofluorescence microscopy with anti-ubiquitin antibodies, and recorded the presence of ubiquitinated proteins in ZP preparations from high-quality metaphase II ova. More recently, they have observed Gly-Gly modifications, a fingerprint of ubiquitinated internal Lys-residues, on all three components of porcine ZP (ZPA, ZPB, and ZPC) using Nanospray LC-MS/MS spectroscopy (Peng et al. 2003; Zimmerman et al. 2011). These findings corroborate the pattern of sequential ubiquitination and proteasomal degradation of the ascidian sperm-receptor protein HrVC70, an analogue of mammalian ZP. Similarly, Yokota and Sawada (2007) reported that the vitelline envelopes of sea urchin eggs are already ubiquitinated before fertilization (Yokota and Sawada 2007).

Enzymatically active proteasomes have been found in the boar sperm acrosome. There is evidence that sperm proteasomes are exposed onto the sperm surface and remain associated with acrosomal membranes during sperm-ZP penetration. Boar sperm proteasomes are associated with acrosomal membranes and matrix before $\mathrm{AE}$ and remain associated with the inner acrosomal membrane after AE (Morales et al. 2004; Zimmerman and Sutovsky 2009; Yi et al. 2009; Yi et al. 2010a, b). Proteasomal activity is present in motile boar spermatozoa (Yi et al. 2009). Adenosine triphosphate (ATP) is essential for the integrity of the $26 \mathrm{~S}$ proteasome, which is maintained by the six 19S ATPase subunits. Depletion of sperm surface ATP by Solanum tuberosum apyrase inhibits sea urchin fertilization (Yokota and Sawada 2007) and porcine in vitro fertilization (IVF) (Yi et al. 2009). Enzymatic activity of boar sperm proteasomes was confirmed in whole, motile spermatozoa, sperm acrosomal fractions, and affinity purified proteasomes (Yi et al. 2009; Miles et al. 2013). These purified proteasomes were also tested for functionality via casein degradation (Manandhar and Sutovsky, unpublished data). 


\subsection{Sperm Proteasomes Can Degrade Zona Pellucida Proteins in Solution and In Situ}

Studies in porcine and avian models suggest that sperm proteasomes can degrade the sperm receptor on porcine $\mathrm{ZP}$ and on the vitelline coat of Japanese quail egg, respectively (Zimmerman et al. 2011; Sasanami et al. 2012). It is difficult to capture the action of sperm acrosomal enzymes during fertilization, but the Sutovsky lab developed an in vitro assay using live, freshly collected, and capacitated boar spermatozoa coincubated with the ZP proteins solubilized from 100 meiotically mature, fertilization-competent porcine oocytes (Zimmerman et al. 2011). The soluble ZP proteins are thus enabled to bind to sperm acrosomal surface as the zona matrix would during fertilization and induce acrosomal exocytosis, resulting in the formation of acrosomal shrouds similar to those seen on the surface of ZP during IVF. Because there is no solid zona matrix in this assay, the acrosomal shrouds are easily separated from spermatozoa and interrogated for the degradation of the bound $\mathrm{ZP}$-proteins. A distinct degradation pattern of the porcine sperm-receptor component ZPC was observed, similar to the degradation pattern of ZPC with purified sperm proteasomes (Zimmerman et al. 2011). The observed, rapid degradation of ZPC by spermatozoa and purified sperm proteasomes was inhibited by ATP depletion with $S$. tuberosum apyrase and with proteasomal inhibitors (MG132, CLBL, and Epoxomicin); it was accelerated by ubiquitin-aldehyde, a C-terminally modified ubiquitin protein that stimulates proteasomal proteolysis (Zimmerman et al. 2011). Furthermore, they were able to record that purified boar sperm proteasomes can digest intact pig ZP of in vitro maturing ova and reduce the rate of polyspermic fertilization after IVF (Zimmerman et al. 2011). These results were corroborated by a study in Japanese quail model that used a similar in vitro system to demonstrate that the sperm proteasome is important for avian fertilization and helps sperm penetration through the perivitelline membrane, which is homologous to the $\mathrm{ZP}$ in mammals. Japanese quail spermatozoa contain proteasomes localized in the acrosomal region and can degrade the ZP1 protein in a fashion similar to the degradation of ZP3/ZPC in the porcine model; this degradation is also inhibited by proteasomal inhibitor MG132 and by extracellular ATP depletion by apyrase (Sasanami et al. 2012).

\subsection{Recent Advances in the Study of Sperm Ubiquitin Proteasome System}

There is a growing acceptance of the involvement of the ubiquitin proteasome system in the reproductive process in plants, ascidians, echinoderms, and mammals, but many outstanding questions remain. How is sperm proteasomal activity regulated during sperm storage, capacitation, AE, and ZP penetration? How are proteasomes inserted in the acrosome during spermiogenesis? What is the role of 
deubiquitinating enzymes in fertilization? Can we target sperm proteasomes for a contraceptive effect? Novel animal models and assays will pave the way to elucidate these mechanisms.

A novel transgenic boar model with green fluorescent protein (GFP) fused to the C-terminus of 20S proteasomal core subunit alpha-type 6 (PSMA1) has been developed through a joint effort between the Sutovsky, Prather, and Wells laboratories at the University of Missouri-Columbia (Miles et al. 2013). Functional GFP-tagged proteasomes have been shown to be incorporated in not only fertilization-competent spermatozoa but in other tissues and cell types (Miles et al. 2013). Using crossimmunoprecipitation experiments, the authors identified various proteins interacting with the GFP-PSMA1 subunit such as lactadherin/MFGE8, spermadhesins, and disintegrins/ADAM metalloproteinases; these proteins may regulate sperm proteasomal activity or may be the substrates of proteasomal proteolysis during fertilization (Miles et al. 2013). They have also proposed a method of isolating enzymatically active GFP proteasomes through GFP affinity purification (Miles et al. 2013). This novel model will be useful for studies of fertilization and wherever UPS plays a role in cellular function or pathology.

A protection assay utilizing proteasomal inhibitors was used as an alternative method of identifying sperm-proteasome interacting proteins. The treatment of porcine spermatozoa with proteasomal inhibitors during coincubation with solubilized ZP proteins led to the accumulation of sperm acrosomal surface-associated proteins that would otherwise be degraded during AE (Zimmerman et al. 2011). The identified proteins that were protected from degradation by proteasomal inhibitors included sperm adhesion molecule 1 (SPAM1), lactadherin/MFGE8, zona pellucida-binding protein 2 (ZPBP2), and fragments of acrosin-binding protein ACRBP (SP32) (Zimmerman et al. 2011). Proteasomal degradation of these acrosomal zona-binding proteins could facilitate the ZP-induced acrosomal exocytosis or serve to terminate primary sperm-ZP binding as the fertilizing spermatozoon starts to move forward and penetrate deeper into the ZP. Furthermore, proteasomal inhibitors suppressed the induction of $\mathrm{AE}$ when human spermatozoa were incubated with recombinant human ZP3 and ZP4 (Chakravarty et al. 2008). Current research also implicates the ubiquitin proteasome system in sperm capacitation and $\mathrm{AE}$, and that these sperm transformations may be regulated and reversed by deubiquitinating enzymes that also regulate oocyte anti-polyspermy defense and oocyte maturation. Inhibition of the ubiquitin-activating enzyme UBA1 (E1) during boar sperm capacitation alters proteasomal subunit properties and sperm-fertilizing ability in vitro in a dose-dependent manner (Yi et al. 2012). Ubiquitin-conjugating enzyme, the ubiquitin-ligase UBR7, has been detected in the boar sperm acrosome, and de novo ubiquitination of UBB+1 has been achieved using sperm acrosomal extract (S.W. Zimmerman and P. Sutovsky, unpublished data). Deubiquitinating enzymes from the ubiquitin C-terminal hydrolase (UCHL) family have been shown to regulate anti-polyspermy defense and oocyte cortex and meiotic spindle formation. Block of sperm UCHL3 increases porcine polyspermy in vitro while supplementation of recombinant UCHs to IVF media reduced polyspermy and increased the rate of monospermic fertilization (Yi et al. 2007b). Interference with bovine oocyte 
UCHL1 alters cortical granule maturation and causes polyspermy (Susor et al. 2010). Block of murine oocyte UCHL1 and UCHL3 prevents sperm incorporation in the ooplasm and causes meiotic spindle anomalies and polar body extrusion defects (Mtango et al. 2012a, b). Subfertility, in vitro polyspermy, and failed morula compaction have been reported in the gad mutant mouse expressing an inactive form of UCHL1 (Kwon et al. 2003; Mtango et al. 2012a). These data suggest that ubiquitination plays a key role in sperm function and that the deubiquitinating enzymes (UCHL1 and UCHL3) are important during fertilization and pre-implantation embryo development.

\subsection{Conclusions}

Accumulating evidence suggests that the putative mammalian egg coat lysin is the $26 \mathrm{~S}$ proteasome. There are data supporting all the prerequisites in the porcine fertilization model: (1) all three pig zona components (ZPA, ZPB, ZPC) are ubiquitinated, (2) enzymatically active proteasomes are present in the boar sperm acrosome and exposed on the acrosomal surface, and (3) motile boar spermatozoa and isolated boar sperm proteasomes are capable of degrading the sperm receptor components of the zona pellucida in vitro.

Recent advances have elucidated more ways in which the UPS affects mammalian reproduction that could possibly be manipulated to develop novel forms of nonhormonal contraceptives. Sperm proteasomes have been shown to copurify with various acrosomal sperm membrane-binding proteins. Sperm capacitation and acrosome reaction have been reported to be altered by interference with UPS enzymes responsible for ubiquitin-substrate conjugation, deubiquitination, and proteasomal proteolysis. Acrosomal extracts can ubiquitinate exogenous substrates. Therefore, the properties of sperm-borne proteasomes are consistent with their proposed role of mammalian zona lysin. Evidence from several laboratories, animal models, and human gamete studies supports the participation of sperm proteasomes in multiple aspects of the fertilization process, including sperm capacitation, sperm zona binding, acrosomal exocytosis, and sperm-zona/vitelline coat penetration.

Acknowledgments We thank Ms. Kathryn Craighead for editorial and clerical assistance. Our gratitude belongs to our colleagues/associates Dr. Young-Joo Yi, Ms. Miriam Sutovsky, Ms. Wonhee Song, Dr. Peter Vargovic, Dr. Gaurishankar Manandhar, and Dr. Shawn Zimmerman, as well as to our collaborators Drs. Randal Prather, Kevin Wells, Richard Oko, Satish Gupta, and David Miller. Some of the work reviewed in this manuscript was supported by Agriculture and Food Research Initiative Competitive Grants no. 2011-67015-20025 and 2013-67015-20961 from the USDA National Institute of Food and Agriculture, and by the seed funding from the Food for the Twenty-first Century Program of the University of Missouri.

Open Access: This article is distributed under the terms of the Creative Commons Attribution Noncommercial License which permits any noncommercial use, distribution, and reproduction in any medium, provided the original author(s) and source are credited. 


\section{References}

Austin CR, Bishop MW (1958) Role of the rodent acrosome and perforatorium in fertilization. Proc R Soc Lond B Biol Sci 149(935):241-248

Baba T, Azuma S et al (1994) Sperm from mice carrying a targeted mutation of the acrosin gene can penetrate the oocyte zona pellucida and effect fertilization. J Biol Chem 269(50): 31845-31849

Baker MA, Reeves G et al (2007) Identification of gene products present in Triton X-100 soluble and insoluble fractions of human spermatozoa lysates using LC-MS/MS analysis. Proteomics Clin Appl 1(5):524-532

Bedard N, Yang Y et al (2011) Mice lacking the USP2 deubiquitinating enzyme have severe male subfertility associated with defects in fertilization and sperm motility. Biol Reprod 85(3):594-604

Bedford JM (1998) Mammalian fertilization misread? sperm penetration of the eutherian zona pellucida is unlikely to be a lytic event. Biol Reprod 59(6):1275-1287

Belote JM, Zhong L (2009) Duplicated proteasome subunit genes in Drosophila and their roles in spermatogenesis. Heredity (Edinb) 103(1):23-31

Bleil JD, Wassarman PM (1980) Mammalian sperm-egg interaction: identification of a glycoprotein in mouse egg zonae pellucidae possessing receptor activity for sperm. Cell 20(3): 873-882

Boavida LC, Vieira AM et al (2005) Gametophyte interaction and sexual reproduction: how plants make a zygote. Int J Dev Biol 49(5-6):615-632

Bohring C, Krause W (2003) Characterization of spermatozoa surface antigens by antisperm antibodies and its influence on acrosomal exocytosis. Am J Reprod Immunol 50(5):411-419

Bohring C, Krause E et al (2001) Isolation and identification of sperm membrane antigens recognized by antisperm antibodies, and their possible role in immunological infertility disease. Mol Hum Reprod 7(2):113-118

Book AJ, Smalle J et al (2009) The RPN5 subunit of the 26s proteasome is essential for gametogenesis, sporophyte development, and complex assembly in Arabidopsis. Plant Cell 21(2): 460-478

Byrne K, Leahy T et al (2012) Comprehensive mapping of the bull sperm surface proteome. Proteomics 12(23-24):3559-3579

Callis J, Bedinger P (1994) Developmentally regulated loss of ubiquitin and ubiquitinated proteins during pollen maturation in maize. Proc Natl Acad Sci USA 91(13):6074-6077

Chakravarty S, Suraj K et al (2005) Baculovirus-expressed recombinant human zona pellucida glycoprotein-B induces acrosomal exocytosis in capacitated spermatozoa in addition to zona pellucida glycoprotein-C. Mol Hum Reprod 11(5):365-372

Chakravarty S, Bansal P et al (2008) Role of proteasomal activity in the induction of acrosomal exocytosis in human spermatozoa. Reprod Biomed Online 16(3):391-400

Chen C, Huang C et al (2008) Subunit-subunit interactions in the human $26 \mathrm{~S}$ proteasome. Proteomics 8(3):508-520

Deveraux Q, van Nocker S et al (1995) Inhibition of ubiquitin-mediated proteolysis by the Arabidopsis 26 S protease subunit S5a. J Biol Chem 270(50):29660-29663

Devoto A, Nieto-Rostro $\mathrm{M}$ et al (2002) COI1 links jasmonate signalling and fertility to the SCF ubiquitin-ligase complex in Arabidopsis. Plant J 32(4):457-466

Diaz ES, Kong M et al (2007) Effect of fibronectin on proteasome activity, acrosome reaction, tyrosine phosphorylation and intracellular calcium concentrations of human sperm. Hum Reprod 22(5):1420-1430

Doelling JH, Phillips AR et al (2007) The ubiquitin-specific protease subfamily UBP3/UBP4 is essential for pollen development and transmission in Arabidopsis. Plant Physiol 145(3): $801-813$

Etlinger JD, Goldberg AL (1977) A soluble ATP-dependent proteolytic system responsible for the degradation of abnormal proteins in reticulocytes. Proc Natl Acad Sci USA 74(1):54-58 
Glickman MH, Ciechanover A (2002) The ubiquitin-proteasome proteolytic pathway: destruction for the sake of construction. Physiol Rev 82(2):373-428

Goldstein G, Scheid M et al (1975) Isolation of a polypeptide that has lymphocyte-differentiating properties and is probably represented universally in living cells. Proc Natl Acad Sci USA 72(1):11-15

Green DP, Purves RD (1984) Mechanical hypothesis of sperm penetration. Biophys J 45(4): 659-662

Gupta SK, Bansal P et al (2009) Human zona pellucida glycoproteins: functional relevance during fertilization. J Reprod Immunol 83(1-2):50-55

Hanna J, Finley D (2007) A proteasome for all occasions. FEBS Lett 581(15):2854-2861

Hershko A (2005) The ubiquitin system for protein degradation and some of its roles in the control of the cell division cycle. Cell Death Differ 12(9):1191-1197

Hershko A, Ciechanover A (1998) The ubiquitin system. Annu Rev Biochem 67:425-479

Honda A, Siruntawineti J et al (2002) Role of acrosomal matrix proteases in sperm-zona pellucida interactions. Hum Reprod Update 8(5):405-412

Ikawa M, Inoue N et al (2010) Fertilization: a sperm's journey to and interaction with the oocyte. J Clin Invest 120(4):984-994

Iwai K (2012) Synthesis and analysis of linear ubiquitin chains. Methods Mol Biol 832:229-238

Johnston DS, Wooters J et al (2005) Analysis of the human sperm proteome. Ann N Y Acad Sci 1061:190-202

Kawano N, Kang W et al (2010) Mice lacking two sperm serine proteases, ACR and PRSS21, are subfertile, but the mutant sperm are infertile in vitro. Biol Reprod 83(3):359-369

Kierszenbaum AL, Rivkin E et al (2011) Cytoskeletal track selection during cargo transport in spermatids is relevant to male fertility. Spermatogenesis 1(3):221-230

Kim ST, Zhang K et al (2006) Exogenous free ubiquitin enhances lily pollen tube adhesion to an in vitro stylar matrix and may facilitate endocytosis of SCA. Plant Physiol 142(4):1397-1411

Kong M, Diaz ES et al (2009) Participation of the human sperm proteasome in the capacitation process and its regulation by protein kinase A and tyrosine kinase. Biol Reprod 80(5): $1026-1035$

Kwon J, Kikuchi T et al (2003) Characterization of the testis in congenitally ubiquitin carboxyterminal hydrolase-1 (Uch-L1) defective (gad) mice. Exp Anim 52(1):1-9

Lin H, Keriel A et al (2000) Divergent N-terminal sequences target an inducible testis deubiquitinating enzyme to distinct subcellular structures. Mol Cell Biol 20(17):6568-6578

Liu CW, Li X et al (2006) ATP binding and ATP hydrolysis play distinct roles in the function of 26S proteasome. Mol Cell 24(1):39-50

Matsumura K, Aketa K (1991) Proteasome (multicatalytic proteinase) of sea urchin sperm and its possible participation in the acrosome reaction. Mol Reprod Dev 29(2):189-199

Miles EL, O'Gorman C, Zhao J, Samuel M, Walters E, Yi YJ, Sutovsky M, Prather RS, Wells K, Sutovsky P (2013) Transgenic pig carrying green fluorescent proteasomes. Proc Natl Acad Sci USA 110(16):6334-6339

Morales P, Kong M et al (2003) Participation of the sperm proteasome in human fertilization. Hum Reprod 18(5):1010-1017

Morales P, Pizarro E et al (2004) Extracellular localization of proteasomes in human sperm. Mol Reprod Dev 68(1):115-124

Mtango NR, Sutovsky M et al (2012a) Essential role of maternal UCHL1 and UCHL3 in fertilization and preimplantation embryo development. J Cell Physiol 227(4):1592-1603

Mtango NR, Sutovsky M et al (2012b) Essential role of ubiquitin C-terminal hydrolases UCHL1 and UCHL3 in mammalian oocyte maturation. J Cell Physiol 227(5):2022-2029

Naz RK, Dhandapani L (2010) Identification of human sperm proteins that interact with human zona pellucida3 (ZP3) using yeast two-hybrid system. J Reprod Immunol 84(1):24-31

Pasten C, Morales P et al (2005) Role of the sperm proteasome during fertilization and gamete interaction in the mouse. Mol Reprod Dev 71(2):209-219

Peng J, Schwartz D et al (2003) A proteomics approach to understanding protein ubiquitination. Nat Biotechnol 21(8):921-926 
Pines J (1994) Cell cycle. Ubiquitin with everything. Nature 371(6500):742-743

Qiao H, Wang H et al (2004) The F-box protein AhSLF-S2 physically interacts with S-RNases that may be inhibited by the ubiquitin/26S proteasome pathway of protein degradation during compatible pollination in Antirrhinum. Plant Cell 16(3):582-595

Redgrove KA, Anderson AL et al (2011) Involvement of multimeric protein complexes in mediating the capacitation-dependent binding of human spermatozoa to homologous zonae pellucidae. Dev Biol 356(2):460-474

Rivkin E, Kierszenbaum AL et al (2009) Rnf19a, a ubiquitin protein ligase, and Psmc3, a component of the $26 \mathrm{~S}$ proteasome, tether to the acrosome membranes and the head-tail coupling apparatus during rat spermatid development. Dev Dyn 238(7):1851-1861

Rosenzweig R, Osmulski PA et al (2008) The central unit within the 19S regulatory particle of the proteasome. Nat Struct Mol Biol 15(6):573-580

Sakai N, Sawada H et al (2003) Extracellular ubiquitin system implicated in fertilization of the ascidian, Halocynthia roretzi: isolation and characterization. Dev Biol 264(1):299-307

Sakai N, Sawada MT et al (2004) Non-traditional roles of ubiquitin-proteasome system in fertilization and gametogenesis. Int J Biochem Cell Biol 36(5):776-784

Sasanami T, Sugiura K et al (2012) Sperm proteasome degrades egg envelope glycoprotein ZP1 during fertilization of Japanese quail (Coturnix japonica). Reproduction 144(4):423-431

Sawada H, Sakai N et al (2002a) Extracellular ubiquitination and proteasome-mediated degradation of the ascidian sperm receptor. Proc Natl Acad Sci USA 99(3):1223-1228

Sawada H, Takahashi Y et al (2002b) Localization and roles in fertilization of sperm proteasomes in the ascidian Halocynthia roretzi. Mol Reprod Dev 62(2):271-276

Shur BD, Rodeheffer C et al (2006) Identification of novel gamete receptors that mediate sperm adhesion to the egg coat. Mol Cell Endocrinol 250(1-2):137-148

Speranza A, Scoccianti V et al (2001) Inhibition of proteasome activity strongly affects kiwifruit pollen germination. Involvement of the ubiquitin/proteasome pathway as a major regulator. Plant Physiol 126(3):1150-1161

$\mathrm{Su}$ YH, Chen SH et al (2005) Tandem mass spectrometry identifies proteins phosphorylated by cyclic AMP-dependent protein kinase when sea urchin sperm undergo the acrosome reaction. Dev Biol 285(1):116-125

Susor A, Liskova L et al (2010) Role of ubiquitin C-terminal hydrolase-L1 in antipolyspermy defense of mammalian oocytes. Biol Reprod 82(6):1151-1161

Sutovsky P (2011) Sperm proteasome and fertilization. Soc Reprod Fertil 142:1-14

Sutovsky P, Moreno R et al (2001) A putative, ubiquitin-dependent mechanism for the recognition and elimination of defective spermatozoa in the mammalian epididymis. J Cell Sci 114(pt 9): $1665-1675$

Sutovsky P, McCauley TC et al (2003) Early degradation of paternal mitochondria in domestic pig (Sus scrofa) is prevented by selective proteasomal inhibitors lactacystin and MG132. Biol Reprod 68(5):1793-1800

Sutovsky P, Manandhar G et al (2004) Proteasomal interference prevents zona pellucida penetration and fertilization in mammals. Biol Reprod 71(5):1625-1637

Tanaka K (2009) The proteasome: overview of structure and functions. Proc Jpn Acad Ser B Phys Biol Sci 85(1):12-36

Verma R, Aravind L et al (2002) Role of Rpn11 metalloprotease in deubiquitination and degradation by the 26S proteasome. Science 298(5593):611-615

Voges D, Zwickl P et al (1999) The 26S proteasome: a molecular machine designed for controlled proteolysis. Annu Rev Biochem 68:1015-1068

Walczak H, Iwai K et al (2012) Generation and physiological roles of linear ubiquitin chains. BMC Biol 10:23

Yamashita M, Honda A et al (2008) Reduced fertility of mouse epididymal sperm lacking Prss21/ Tesp5 is rescued by sperm exposure to uterine microenvironment. Genes Cells 13(10): 1001-1013

Yanagimachi R (1994) Fertility of mammalian spermatozoa: its development and relativity. Zygote 2(4):371-372 
Yi YJ, Manandhar G et al (2007a) Mechanism of sperm-zona pellucida penetration during mammalian fertilization: $26 \mathrm{~S}$ proteasome as a candidate egg coat lysin. Soc Reprod Fertil Suppl 63:385-408

Yi YJ, Manandhar G et al (2007b) Ubiquitin C-terminal hydrolase-activity is involved in sperm acrosomal function and anti-polyspermy defense during porcine fertilization. Biol Reprod 77(5):780-793

Yi YJ, Park CS et al (2009) Sperm-surface ATP in boar spermatozoa is required for fertilization: relevance to sperm proteasomal function. Syst Biol Reprod Med 55(2):85-96

Yi YJ, Manandhar G et al (2010a) Inhibition of 19S proteasomal regulatory complex subunit PSMD8 increases polyspermy during porcine fertilization in vitro. J Reprod Immunol 84(2): $154-163$

Yi YJ, Manandhar G et al (2010b) Interference with the 19S proteasomal regulatory complex subunit PSMD4 on the sperm surface inhibits sperm-zona pellucida penetration during porcine fertilization. Cell Tissue Res 341(2):325-340

Yi YJ, Zimmerman SW et al (2012) Ubiquitin-activating enzyme (UBA1) is required for sperm capacitation, acrosomal exocytosis and sperm-egg coat penetration during porcine fertilization. Int J Androl 35(2): 196-210

Yokota N, Sawada H (2007) Sperm proteasomes are responsible for the acrosome reaction and sperm penetration of the vitelline envelope during fertilization of the sea urchin Pseudocentrotus depressus. Dev Biol 308(1):222-231

Yokota N, Kataoka Y et al (2011) Sperm-specific C-terminal processing of the proteasome PSMA1/alpha6 subunit. Biochem Biophys Res Commun 410(4):809-815

Yurewicz EC, Sacco AG et al (1998) Hetero-oligomerization-dependent binding of pig oocyte zona pellucida glycoproteins ZPB and ZPC to boar sperm membrane vesicles. J Biol Chem 273(13):7488-7494

Zhang F, Hu Y et al (2007) Proteasome function is regulated by cyclic AMP-dependent protein kinase through phosphorylation of Rpt6. J Biol Chem 282(31):22460-22471

Zimmerman S, Sutovsky P (2009) The sperm proteasome during sperm capacitation and fertilization. J Reprod Immunol 83(1-2):19-25

Zimmerman SW, Manandhar G et al (2011) Sperm proteasomes degrade sperm receptor on the egg zona pellucida during mammalian fertilization. PLoS One 6(2):e17256 